\title{
Hypothermia Following Spinal Anesthesia in an Infant: Potential Impact of Intravenous Dexmedetomidine and Intrathecal Clonidine
}

\author{
Jordan Arends $^{\mathrm{a}, \mathrm{b}}$, Joseph D. Tobias ${ }^{\mathrm{b}, \mathrm{c}, \mathrm{d}}$
}

\begin{abstract}
The $\alpha_{2}$-adrenergic agonists (dexmedetomidine and clonidine) have been used in several different clinical scenarios in infants and children including sedation during mechanical ventilation, procedural sedation, supplementation of postoperative analgesia, prevention of emergence delirium, control of post-anesthesia shivering, treatment of withdrawal and prolonging of duration of neuraxial anesthesia. Hemodynamic effects including bradycardia and hypotension remain the predominant adverse effects reported with the $\alpha_{2}$-adrenergic agonists. We report hypothermia following intravenous sedation with dexmedetomidine and spinal anesthesia with a combination of bupivacaine and clonidine in a 2 -month-old infant. The potential mechanisms involved are reviewed, the causal relationship between hypothermia and $\alpha_{2}$-adrenergic agonists is explored and interventions to avoid its development are presented.
\end{abstract}

Keywords: Dexmedetomidine; Clonidine; Hypothermia; Spinal anesthesia

\section{Introduction}

The $\alpha_{2}$-adrenergic agonists (dexmedetomidine and clonidine) have been used successfully in infants and children for sedation during mechanical ventilation, to provide procedural sedation, supplement postoperative analgesia, prevent emergence delirium, control post-anesthesia shivering, treat withdrawal and to augment neuraxial anesthesia. Dexmedetomi-

Manuscript submitted November 12, 2019, accepted November 16, 2019

aOhio University Heritage College of Osteopathic Medicine, Dublin, OH, USA

bDepartment of Anesthesiology \& Pain Medicine, Nationwide Children's Hospital, Columbus, OH, USA

'Department of Anesthesiology \& Pain Medicine, The Ohio State University College of Medicine, Columbus, OH, USA

${ }^{\mathrm{d} C}$ Corresponding Author: Joseph D. Tobias, Department of Anesthesiology \& Pain Medicine, Nationwide Children's Hospital, 700 Children's Drive, Columbus, OH 43205, USA. Email: Joseph.Tobias@Nationwidechildrens.org

doi: https://doi.org/10.14740/jmc3391 dine is an $\alpha_{2}$-adrenergic agonist which was first approved by the Food and Drug Administration (FDA) of the United States in 1999 for the sedation of adults during mechanical ventilation and subsequently in 2009 for monitored anesthesia care (MAC) of adults during perioperative care. Like clonidine, dexmedetomidine is a member of the imidazole subclass of the $\alpha_{2}$-adrenergic agonists. Its $\alpha_{2} / \alpha_{1}$ specificity is $1,620: 1$, higher than that of clonidine $(220: 1)$, thereby making it a complete agonist [1].

Two of the more common clinical applications of these agents in our clinical practice include the use of intravenous dexmedetomidine to provide sedation and anxiolysis during awake regional anesthesia as well as the addition of clonidine to a local anesthetic agent to prolong the duration of spinal anesthesia [1-3]. To date, the most frequently cited adverse effects of these agents have included bradycardia and hypotension. We present the development of hypothermia following sedation with dexmedetomidine during spinal anesthesia with a combination of bupivacaine and clonidine in a 2-month-old infant during urological surgery. The potential mechanisms involved are reviewed, the causal relationship with the $\alpha_{2}$ adrenergic agonists is explored and interventions to avoid its development are presented.

\section{Case Report}

Institutional Review Board approval is not required for review and presentation of isolated case reports from Nationwide Children's Hospital (Columbus, Ohio). A 2-month-old, former term, $5.6 \mathrm{~kg}$ infant presented for anesthetic care during cystoscopy and resection of a right ectopic congenital ureterocele. Past history included hydronephrosis and recurrent urinary tract infections. There was no prior surgical history and no known allergies. Medications included cefdinir oral suspension (once daily) and cholecalciferol (two drops every morning). The physical examination was non-contributory and the vital signs were unremarkable. Based on our routine clinical practice, spinal anesthesia was offered and informed consent was obtained from the parents. The patient was transported to the operating room and routine American Society of Anesthesiologists' monitors were placed. Continuous temperature monitoring was not used based on our usual clinical practice during spinal anesthesia. To maintain normothermia, before 
the infant arrived in the operating room, the room was warmed and maintained throughout the procedure. Overhead warming lights were placed when the patient was uncovered while spinal anesthesia was placed and peripheral intravenous access was obtained. Additionally, a forced air warming blanket at $43{ }^{\circ} \mathrm{C}$ was placed under the patient's legs, body and torso and the patient was covered with plastic as allowed by the surgical procedure.

After the lumbar area was cleansed with an antiseptic solution, spinal anesthesia was placed using a 22-gauge spinal needle with a stylet inserted at the L3-4 interspace. Once free flow of cerebrospinal fluid was obtained, $1 \mathrm{~mL}$ of $0.5 \%$ bupivacaine $(5 \mathrm{mg})$ with 1:200,000 epinephrine and clonidine $(5 \mu \mathrm{g})$ was injected. After adequate sensory and motor blockade were obtained, a peripheral intravenous cannula was placed in the lower extremity. Sedation during the procedure was provided by dexmedetomidine (two $2-\mu \mathrm{g}$ doses) and propofol (2 mg). No change in vital signs was noted after placement of the spinal anesthesia or the administration of dexmedetomidine and propofol. The surgical procedure lasted approximately $25 \mathrm{~min}$. No additional sedation or analgesia was provided. The patient was transported to the post-anesthesia care unit (PACU). On arrival to the PACU, vital signs were stable and the body temperature was $36.4^{\circ} \mathrm{C}$. After approximately $30 \mathrm{~min}$, the patient was transferred to phase 2 recovery. Shortly after arrival, the patient was noted to be pale and lethargic. Vital signs included temperature (rectal) $35.0^{\circ} \mathrm{C}$, heart rate 110 beats $/ \mathrm{min}$, respirations 24 breaths/min with 10 - $15 \mathrm{~s}$ pauses between breaths and longer periods of apnea that required stimulation, blood pressure $89 / 51 \mathrm{~mm} \mathrm{Hg}$ and oxygen saturation $\left(\mathrm{SpO}_{2}\right)$ 99\%. Serum glucose and hemoglobin were $113 \mathrm{mg} / \mathrm{dL}$ and $9.2 \mathrm{~g} / \mathrm{dL}$, respectively. The patient was transferred back to phase 1 recovery and external warming was initiated. The apnea responded to gentle stimulation and the patient moved all extremities appropriately, but he remained lethargic. With no improvement in the lethargy after an hour, it was decided to admit the patient to the pediatric ICU for ongoing monitoring. Blood cultures were drawn and broad spectrum antibiotics were started. At the time of admission to the pediatric ICU, the patient's body temperature was $36.4{ }^{\circ} \mathrm{C}$, pulse 142 beats/min, respirations 31 breaths/ min, blood pressure 90/43 mm $\mathrm{Hg}$ and $\mathrm{SpO}_{2}$ 100\%. The patient's postoperative course was unremarkable. He was able to maintain normothermia without external warming. Blood cultures were negative, and the antibiotics were discontinued after $48 \mathrm{~h}$ and he was discharged home. Follow-up visit with the pediatric urologist was unremarkable.

\section{Discussion}

Data from animal and retrospective epidemiological studies have suggested the potential for long-term neurocognitive effects from various anesthetic agents on the developing brain $[4,5]$. Although there remains no clear evidence of neurocognitive effects from prospective studies in infants and children, the United States FDA has issued a warning of possible adverse effects of repeated or lengthy use of general anesthesia or sedation during procedures in children less than 3 years of age [6]. To avoid these potential risks, our institution has started a program which offers regional anesthesia instead of general anesthesia for lower abdominal, perineal or lower extremity surgery [7].

When neuraxial anesthesia is used instead of general anesthesia, our clinical practice includes the use of dexmedetomidine to provide sedation. Dexmedetomidine is chosen as animal data have suggested that its potential pro-apoptotic effect is limited unlike other anesthetic agents (benzodiazepines, barbiturates, propofol or the volatile anesthetic agents) [8, 9]. Additionally, clonidine is frequently added to the spinal anesthesia solution to prolong the duration of surgical anesthesia $[3,10]$. Hemodynamic effects including bradycardia and hypotension remain the predominant adverse effects reported with the $\alpha_{2}$-adrenergic agonists [11]. However, temperature control and thermogenesis may also be affected by several potential mechanisms. Direct activation of $\alpha_{2}$-adrenergic receptors in the hypothalamus decreases metabolic heat production [12]. The vasoconstrictive response to hypothermia is blunted thereby allowing for ongoing vasodilatation and heat loss as core body temperature decreases [13]. In the periphery, activation of post-synaptic $\alpha_{2}$-adrenergic receptors inhibits lipolysis and non-shivering thermogenesis in brown fat $[14,15]$. The physiologic effects of $\alpha_{2}$-adrenergic agonists, predominantly dexmedetomidine, on temperature regulation and heat generation have led to its use to prevent shivering following anesthetic care and during therapeutic hypothermia [16-18].

Although rarely reported as an adverse effect, as illustrated by our patient and a previous report from the literature, these physiologic effects may lead to hypothermia especially in neonates and young infants. Finkel et al reported hypothermia and bradycardia in a 2-day-old, 3-kg infant when dexmedetomidine was used for sedation and to provide immobility following repair of bladder exstrophy [19]. Hypothermia developed approximately $9 \mathrm{~h}$ postoperatively with a dexmedetomidine infusion at $0.4 \mu \mathrm{g} / \mathrm{kg} / \mathrm{h}$ and an epidural infusion of ropivacaine and fentanyl. The hypothermia was corrected by the placement of an overhead radiant warmer and a decrease of the dexmedetomidine infusion to $0.2 \mu \mathrm{g} / \mathrm{kg} / \mathrm{h}$. Additional evidence for the potential impact of $\alpha_{2}$-adrenergic agonists on thermoregulation and their potential role in the development of hypothermia is provided by animal data [20-22].

In infants, the normal response to hypothermia initially includes vasoconstriction and increased metabolic heat production via non-shivering thermogenesis. These actions may be blocked by the stimulation of central and peripheral $\alpha_{2}$ adrenergic receptors. We would postulate that these effects may occur regardless of the route (intravenous or neuraxial) of administration of the $\alpha_{2}$-adrenergic agonist. In our patient, it is not possible to absolutely prove whether the intravenous dexmedetomidine or the intrathecal clonidine was primarily responsible for our patient's hypothermia. However, other causes of hypothermia and lethargy including exposure, sepsis and hypoglycemia were ruled out. Although spinal anesthesia with local anesthetic agents may have contributed to the inability to respond to hypothermia by blocking thermogenesis, thermoregulation has been shown to be intact during epidural anesthesia in adults and in young children receiving caudal anesthesia with general anesthesia [23-25].

When $\alpha_{2}$-adrenergic agonists are administered during the 
perioperative period to neonates and infants, we would recommend continuous monitoring of core body temperature intraoperatively even when spinal anesthesia is performed. Although continuous temperature monitoring is considered the standard of care during general anesthesia, it is not commonplace during the clinical practice of spinal instead of general anesthesia. Active warming devices (forced air warming, increased environmental temperature and external heating lamps) should be routinely used to maintain normothermia. The hypothermia in our patient was ultimately attributed to the $\alpha_{2}$-adrenergic agonists; however, other conditions including sepsis may result in bradycardia, temperature instability, hypotension and apnea in neonates and infants. Therefore, the potential for such etiologies must be investigated and treatment should be initiated until they are ruled out.

\section{Acknowledgments}

None to declare.

\section{Financial Disclosure}

None to declare.

\section{Conflict of Interest}

None to declare.

\section{Informed Consent}

Institutional Review Board approval is not required for review and presentation of isolated case reports from Nationwide Children's Hospital (Columbus, Ohio).

\section{Author Contributions}

Jordan Arends: review of case, preparation of initial drafts and final version. Joseph Tobias: concept, review of drafts and approval of final version.

\section{References}

1. Tobias JD. Dexmedetomidine: applications in pediatric critical care and pediatric anesthesiology. Pediatr Crit Care Med. 2007;8(2):115-131.

2. Shutes BL, Gee SW, Sargel CL, Fink KA, Tobias JD. Dexmedetomidine as Single Continuous Sedative During Noninvasive Ventilation: Typical Usage, Hemodynamic Effects, and Withdrawal. Pediatr Crit Care Med. 2018;19(4):287-297.

3. Trifa M, Tumin D, Whitaker EE, Bhalla T, Jayanthi VR, Tobias JD. Spinal anesthesia for surgery longer than 60 min in infants: experience from the first 2 years of a spinal anesthesia program. J Anesth. 2018;32(4):637-640.

4. Blaylock M, Engelhardt T, Bissonnette B. Fundamentals of neuronal apoptosis relevant to pediatric anesthesia. Paediatr Anaesth. 2010;20(5):383-395.

5. Sun L. Early childhood general anaesthesia exposure and neurocognitive development. Br J Anaesth. 2010;105(Suppl 1):i61-68.

6. Davidson AJ, Sun LS. Clinical Evidence for Any Effect of Anesthesia on the Developing Brain. Anesthesiology. 2018;128(4):840-853.

7. Whitaker EE, Wiemann BZ, DaJusta DG, Alpert SA, Ching CB, McLeod DJ, Tobias JD, et al. Spinal anesthesia for pediatric urological surgery: Reducing the theoretic neurotoxic effects of general anesthesia. J Pediatr Urol. 2017;13(4):396-400.

8. Wei Y, Hu J, Liang Y, Zhong Y, He D, Qin Y, Li L, et al. Dexmedetomidine pretreatment attenuates propofolinduced neurotoxicity in neuronal cultures from the rat hippocampus. Mol Med Rep. 2016;14(4):3413-3420.

9. Wang $X$, Zhao B, Li X. Dexmedetomidine attenuates isoflurane-induced cognitive impairment through antioxidant, anti-inflammatory and anti-apoptosis in aging rat. Int J Clin Exp Med. 2015;8(10):17281-17288.

10. Rochette A, Troncin R, Raux O, Dadure C, Lubrano JF, Barbotte E, Capdevila X. Clonidine added to bupivacaine in neonatal spinal anesthesia: a prospective comparison in 124 preterm and term infants. Paediatr Anaesth. 2005;15(12):1072-1077.

11. Lam F, Bhutta AT, Tobias JD, Gossett JM, Morales L, Gupta P. Hemodynamic effects of dexmedetomidine in critically ill neonates and infants with heart disease. Pediatr Cardiol. 2012;33(7):1069-1077.

12. Lahdesmaki J, Sallinen J, MacDonald E, Sirvio J, Scheinin M. Alpha2-adrenergic drug effects on brain monoamines, locomotion, and body temperature are largely abolished in mice lacking the alpha2A-adrenoceptor subtype. Neuropharmacology. 2003;44(7):882-892.

13. Talke P, Tayefeh F, Sessler DI, Jeffrey R, Noursalehi M, Richardson C. Dexmedetomidine does not alter the sweating threshold, but comparably and linearly decreases the vasoconstriction and shivering thresholds. Anesthesiology. 1997;87(4):835-841

14. Dawkins MJ, Scopes JW. Non-shivering thermogenesis and brown adipose tissue in the human new-born infant. Nature. 1965;206(980):201-202.

15. Sinclair JC. Thermal control in premature infants. Annu Rev Med. 1972;23:129-148.

16. Bicer C, Esmaoglu A, Akin A, Boyaci A. Dexmedetomidine and meperidine prevent postanaesthetic shivering. Eur J Anaesthesiol. 2006;23(2):149-153.

17. Blaine Easley R, Brady KM, Tobias JD. Dexmedetomidine for the treatment of postanesthesia shivering in children. Paediatr Anaesth. 2007;17(4):341-346.

18. O'Mara K, Weiss MD. Dexmedetomidine for sedation of neonates with HIE undergoing therapeutic hypothermia: a single-center experience. AJP Rep. 2018;8(3):e168e173.

19. Finkel JC, Quezado ZM. Hypothermia-induced bradycar- 
dia in a neonate receiving dexmedetomidine. J Clin Anesth. 2007;19(4):290-292.

20. Lu J, Liu LJ, Zhu JL, Shen Y, Zhuang ZW, Zhu CL. Hypothermic properties of dexmedetomidine provide neuroprotection in rats following cerebral ischemia-reperfusion injury. Exp Ther Med. 2019;18(1):817-825.

21. McAdams RM, McPherson RJ, Kapur R, Phillips B, Shen DD, Juul SE. Dexmedetomidine reduces cranial temperature in hypothermic neonatal rats. Pediatr Res. 2015;77(6):772-778.

22. Lin MT, Shian LR, Leu SY. Clonidine-induced hypothermia: possible involvement of cholinergic and serotoner- gic mechanisms. Naunyn Schmiedebergs Arch Pharmacol. 1984;326(2):124-128.

23. Sessler DI, Ponte J. Shivering during epidural anesthesia. Anesthesiology. 1990;72(5):816-821.

24. Bissonnette B, Sessler DI. Thermoregulatory thresholds for vasoconstriction in pediatric patients anesthetized with halothane or halothane and caudal bupivacaine. Anesthesiology. 1992;76(3):387-392.

25. Bissonnette B, Sessler DI. The thermoregulatory threshold in infants and children anesthetized with isoflurane and caudal bupivacaine. Anesthesiology. 1990;73(6):11141118 . 